\title{
Short-Term Results of Transforaminal Lumbar Interbody Fusion Using Pedicle Screw with Cortical Bone Trajectory Compared with Conventional Trajectory
}

\author{
Yuji Kasukawa, Naohisa Miyakoshi, Michio Hongo, \\ Yoshinori Ishikawa, Daisuke Kudo, Yoichi Shimada \\ Department of Orthopedic Surgery, Akita University Graduate School of Medicine, Akita, Japan
}

\begin{abstract}
Study Design: Case-control study.
Purpose: To evaluate clinical and radiological results of transforaminal lumbar interbody fusion (TLIF) performed with cortical bone trajectory (CBT) pedicle screw insertion with those of TLIF using 'conventional' or percutaneous pedicle screw insertion.

Overview of Literature: CBT is a new trajectory for pedicle screw insertion in the lumbar spine; clinical and radiological results of TLIF using pedicle screws inserted with CBT are unclear.

Methods: In total, 26 patients (11 males, 15 females) were enrolled in this retrospective study and divided into three groups: TLIF with pedicle screw insertion by conventional minimally invasive methods via the Wiltse approach (M-TLIF, n=10), TLIF with percutaneous pedicle screw insertion (P-TLIF, n=6), and TLIF with pedicle screw insertion with CBT (CBT-TLIF, $n=10)$. Surgical results and preand postoperative radiological findings were evaluated and compared.

Results: Intraoperative blood loss was significantly less with CBT-TLIF ( $p=0.03)$ than with M-TLIF. Postoperative lordotic angles did not differ significantly among the three groups. Complete fusions were obtained in 10 of 12 levels (83\%) with M-TLIF, in seven levels $(100 \%)$ with P-TLIF, and in 10 of 11 levels (91\%) with CBT-TLIF. On postoperative computed tomography, correct positioning was seen in $84.1 \%$ of M-TLIF screws, $88.5 \%$ of P-TLIF screws, and $90 \%$ of CBT-TLIF screws.

Conclusions: CBT-TLIF resulted in less blood loss and a shorter operative duration than M-TLIF or P-TLIF. Postoperative rates of bone union, maintenance of lordotic angles, and accuracy of pedicle screw positions were similar among the three groups.
\end{abstract}

Keywords: Transforaminal lumbar interbody fusion; Cortical bone trajectory; Conventional trajectory; Percutaneous insertion; Computed tomography

\section{Introduction}

The clinical results of transforaminal lumbar interbody fusion (TLIF) have been favorable for degenerative spon- dylolisthesis, kyphoscoliosis, and instability of the lumbar spine $[1,2]$. However, there has been concern regarding pedicle screw placement during TLIF. Exposure lateral to the facet joint to insert a pedicle screw requires a rela-

Received Nov 2, 2014; Accepted Nov 8, 2014

Corresponding author: Yuji Kasukawa

Department of Orthopedic Surgery, Akita University Graduate School of Medicine,

1-1-1 Hondo, Akita 010-8543, Japan

Tel: +81-18-884-6148, Fax: +81-18-836-2617, E-mail: kasukawa@doc.med.akita-u.ac.jp 
tively long incision and muscle dissection, which may be related to postoperative low back pain from injury to the posteromedial branch of the nerve root crossing the facet joint and damage to the exposed and retracted back musculature. To minimize the incision and muscle dissection and thus reduce these problems, TLIF with minimally invasive pedicle screw insertion (M-TLIF) [3] and TLIF with percutaneous pedicle screw insertion (P-TLIF) [4] have been developed. However, several clinical concerns, such as low back pain, learning curve, radiation exposure, and incorrect pedicle screw placement, have also been associated with M-TLIF and P-TLIF [5-7].

A new trajectory for pedicle screw insertion of pedicle screw placement, the cortical bone trajectory (CBT), was reported by Santoni et al. [8] in 2009 and may address these problems. The new trajectory was from medial to lateral and cranial to caudal; this does not require wide exposure of the back muscle and thus reduces operative invasion compared with conventional or percutaneous pedicle screw insertion. However, the differences in operative invasion, accuracy of pedicle screw insertion, and postoperative fusion rate between TLIF with CBT (CBTTLIF) and other methods of pedicle screw placement, such as M-TLIF and P-TLIF, remain unknown. In this study, we compared the clinical and radiological results of CBT-TLIF with those of M-TLIF and P-TLIF.

\section{Materials and Methods}

\section{Patients}

In total, 26 patients ( 11 males, 15 females; mean age, 67 years; range, 34-80 years) who underwent TLIF from April 2011 to February 2013 at our hospital were enrolled in this retrospective study. The indications for TLIF were Meyerding grade I or II spondylolisthesis [9] or intraforaminal to lateral disc herniation. We performed three different methods of pedicle screw insertion depending on the time period. From April to November 2011, pedicle screws were placed minimally invasively via lateral inter-muscular Wiltse approach (M-TLIF, $\mathrm{n}=10 ; 6$ males, 4 females; mean age, 63 years). From December 2011 to October 2012, pedicle screws were inserted using a percutaneous system (P-TLIF; $\mathrm{n}=6,2$ males, 4 females; mean age, 71 years). From November 2012 to February 2013, pedicle screws were placed with CBT (CBT-TLIF; $n=10,3$ males, 7 females; mean age, 67 years).

\section{Surgical procedures}

M-TLIF was performed as follows. A unilateral facetectomy was performed at the location of the symptoms to expose the intervertebral foramen via a $6-\mathrm{cm}$ incision. A thorough discectomy was completed and the disc space was filled with local bone graft material and an appropriate parallel Devex cage (DePuy Spine, Raynham, MA, USA) was placed. Open conventional pedicle screws were placed using the Expedium Spine System (DePuy Spine) through a bilateral Wiltse approach. Under fluoroscopic guidance in a perfect posteroanterior projection, a pedicle probe was introduced into the pedicle at a $30^{\circ}$ medial angle and the pedicle was tapped for a screw, taking care not to penetrate the medial wall. A feeler was used to identify breakage of the cortical pedicle walls, and a pedicle screw of appropriate length, as assessed on computed tomography (CT) images, was inserted. The lengths of screws were 40 or $45 \mathrm{~mm}$ and 6.0 or $7.0 \mathrm{~mm}$ in diameter. Finally, under a lateral fluoroscopic view, the length and craniocaudal direction of the screws were checked (Fig. 1).

P-TLIF was performed using the Viper MIS Spine System (DePuy Spine). Following decompression of the affected site and placement of a cage into the disc space via a $6-\mathrm{cm}$ skin incision, the targeting needle was placed on the superolateral border of the pedicle under fluoroscopy via another fascia incision created $1 \mathrm{~cm}$ lateral to the midline skin incision. The targeting needle was introduced into the pedicle under posteroanterior and lateral fluoroscopic visualization. The targeting needle was replaced with a $\mathrm{K}$ wire, and a screw with an extended sleeve was then placed over the $\mathrm{K}$ wire and inserted into the vertebral body after tapping. Pre-bent rods were placed bilaterally using the Viper system and fixed with compressive force at the facetectomy side (Fig. 2).

CBT-TLIF was performed using the CD HORIZON SOLERA Spinal System $4.75 \mathrm{~mm}$ (Medtronic, Memphis TN, USA). After exposure of the surgical field, an entry point for insertion of the CBT screw was drilled in the medio-caudal side of the pedicle with a $2 \mathrm{~mm}$-diameter air drill under fluoroscopic guidance. A straight probe was used to create a trajectory for the CBT screw from the entry point to the opposite corner of the pedicle and vertebral body under anteroposterior fluoroscopic guidance. A short L-shaped $\mathrm{K}$ wire was placed to mark the trajectory. Decompression and cage placement were performed in the same fashion as in M-TLIF and P-TLIF. After cage 

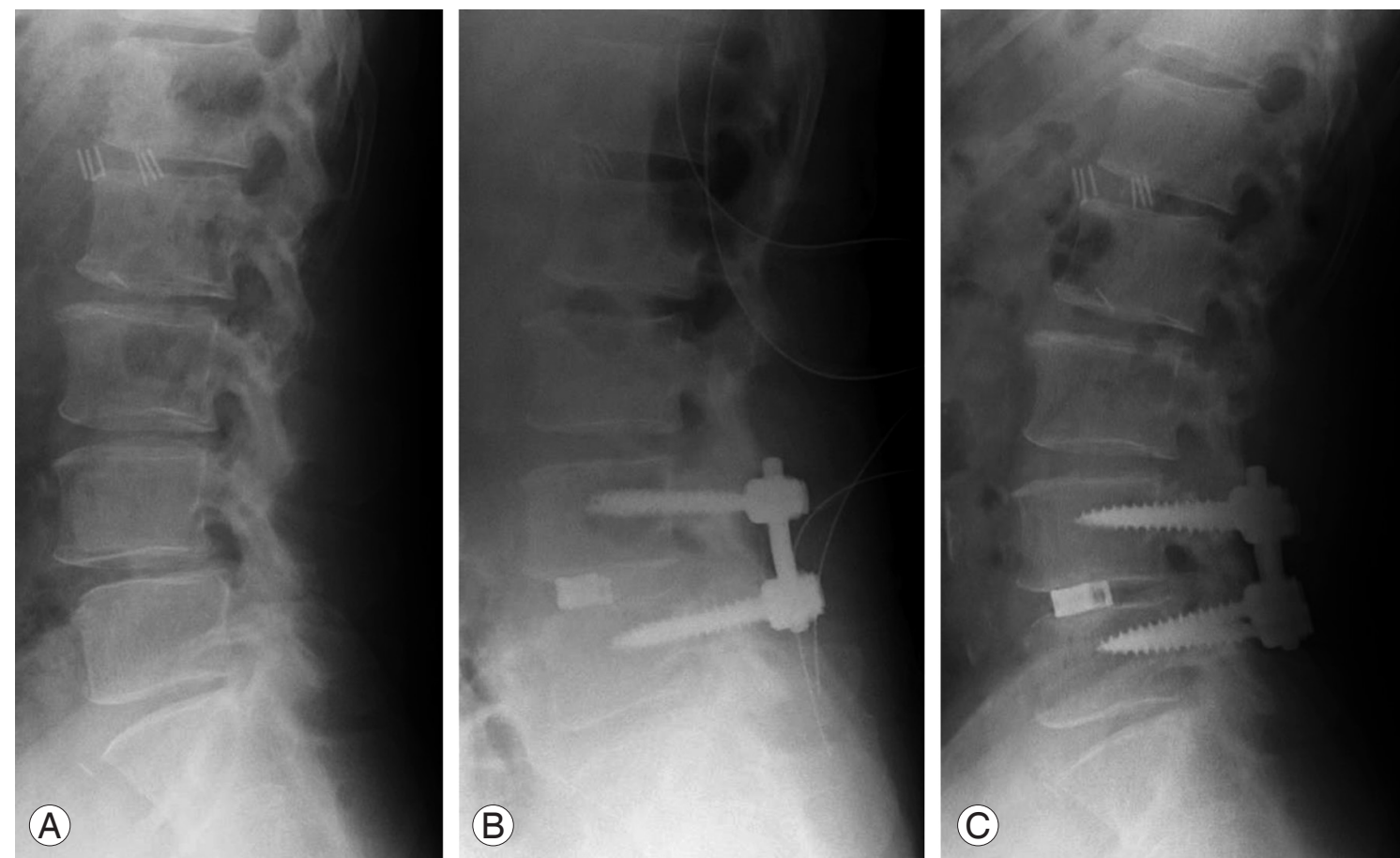

Fig. 1. Lateral radiographs of M-TLIF. (A) Preoperative radiograph, (B) postoperative radiograph, (C) radiograph at final follow-up. M-TLIF, transforaminal lumbar interbody fusion with minimally invasive pedicle screw insertion.
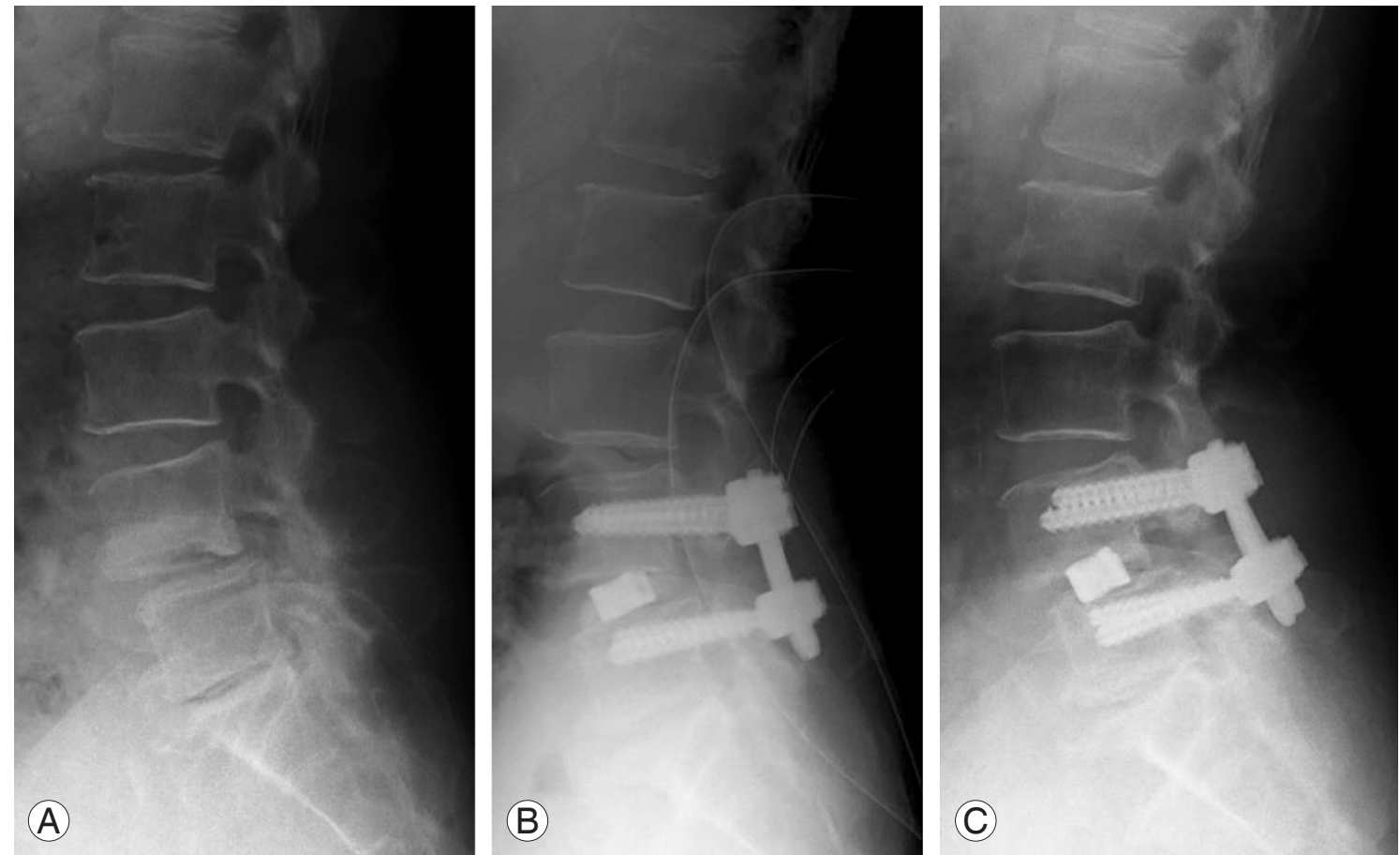

Fig. 2. Lateral radiographs of P-TLIF. Preoperative radiograph (A), postoperative radiograph (B), and radiograph at final follow-up (C). P-TLIF, transforaminal lumbar interbody fusion with percutaneous pedicle screw insertion.

placement, we tapped a hole with successive 4.0-, 4.5-, and 5.5-mm taps targeted to the posterior one-third of the vertebral body. When the tap reached the endosteal cortex of the vertebral body under lateral fluoroscopic guid- 
Table 1. Patient characteristics and surgical parameters

\begin{tabular}{lccc}
\hline Characteristic & M-TLIF & P-TLIF & CBT-TLIF \\
\hline No. of case & 10 & 6 & 10 \\
\hline Gender (male:female) & $6: 4$ & $2: 4$ & $3: 7$ \\
\hline Diseases & 7 & 5 & 8 \\
\hline Spondylolisthesis & 3 & 1 & 2 \\
\hline Foraminal hernia & & 5 & 9 \\
\hline Fusion levels & 8 & 0 & 0 \\
\hline One & 2 & 4 & 9 \\
\hline L3-4 & 5 & 1 & 0 \\
\hline L4-5 & 1 & 1 & 1 \\
\hline L5-S & 2 & 13 & 8 \\
\hline Two (L3-4/4-5) & 16 & 5 & \\
\hline Follow-up period (mo) & & \\
\hline
\end{tabular}

M-TLIF, transforaminal lumbar interbody fusion with minimally invasive pedicle screw insertion; P-TLIF, transforaminal lumbar interbody fusion with percutaneous pedicle screw insertion; CBT-TLIF, transforaminal lumbar interbody fusion with pedicle screw insertion with cortical bone trajectory.

ance, screw length was determined. We then inserted 5.5$\mathrm{mm}$ screws from 30 to $40 \mathrm{~mm}$ in length into the hole and placed the rods (Fig. 3).

\section{Diagnoses and surgical levels}

Diagnoses at operation (degenerative spondylolisthesis and foraminal stenosis or hernia), surgical levels, and their distributions among types of pedicle screw placement are presented in Table 1.

\section{Evaluations}

Patient age, gender, body mass index, bone mineral den-
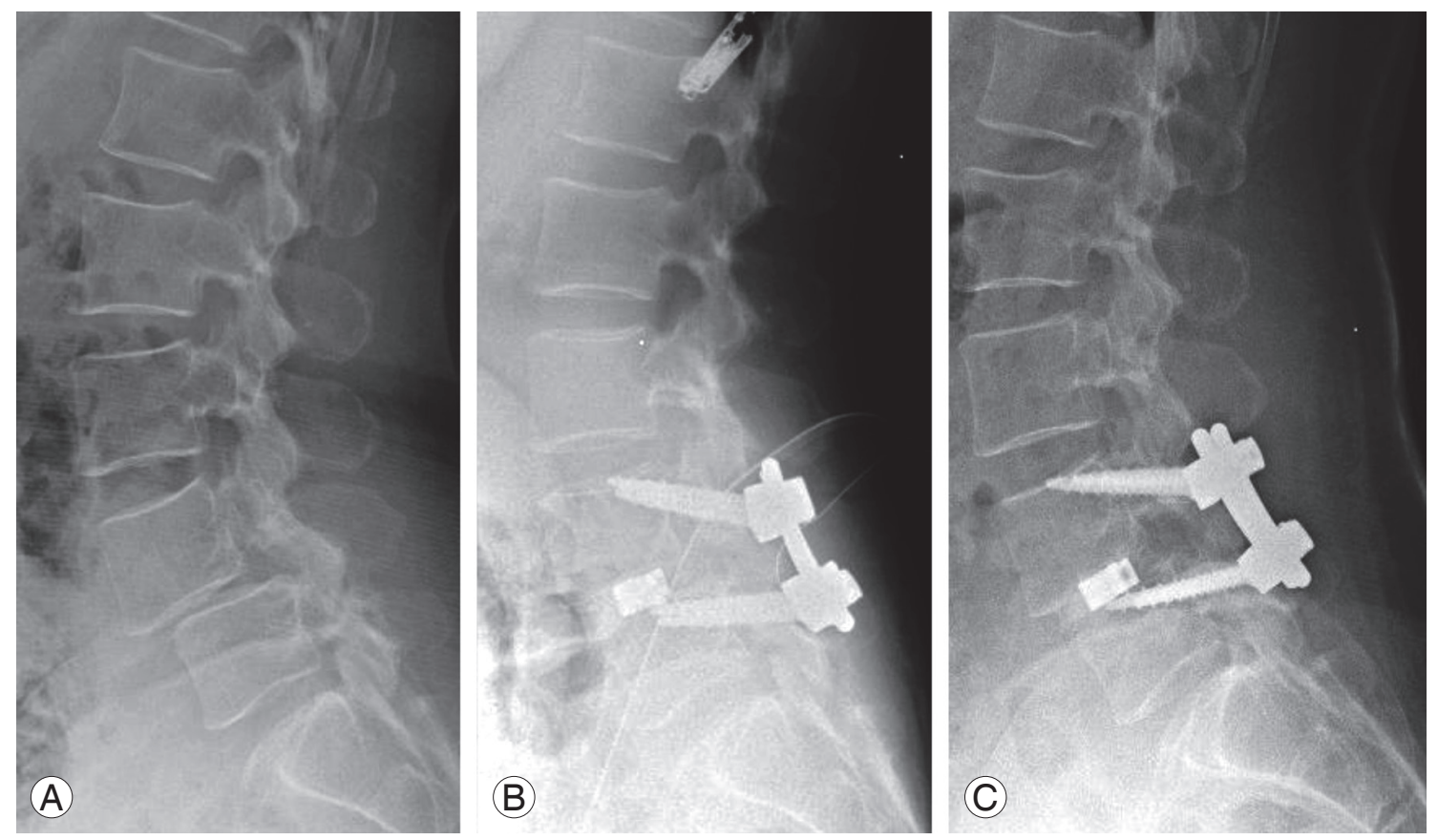

Fig. 3. Lateral radiographs of CBT-TLIF. (A) Preoperative radiograph, (B) postoperative radiograph, and (C) radiograph at final follow-up. CBT-TLIF, transforaminal lumbar interbody fusion with pedicle screw insertion with cortical bone trajectory. 
sity, diagnosis, duration of operation, estimated blood loss (EBL), intraoperative complications, level of fusion, approach of pedicle screw insertion, and radiological findings were obtained from medical records and plain radiographs. Operative duration, EBL during operation, and lordotic angle of fusion levels were evaluated and complications during operation recorded. The lordotic angle-i.e., the angle between the cranial end of the upper vertebra and the caudal end of the lower vertebra (but the cranial end of the S1 vertebra) of the fusion levelwas measured preoperatively and postoperatively and at final follow-up. Bone union at final follow-up was also evaluated on plain radiographs, including flexion and ex- tension lateral images. Definitive fusion was identified by formation of trabecular bony bridges between contiguous vertebral bodies at the instrumented levels and less than $4^{\circ}$ of segmental movement [10]. CT was performed in all patients to check the postoperative positions of screws. The examinations were performed from 2 weeks to 1 year after the operation. The positions of screws were evaluated according to the criteria of Learch et al. [11]. Pedicles were considered to be 'correct' if screws were centered in the pedicle (Fig. 4A, C). We evaluated screws that were in contact with the medial or lateral pedicle wall (Fig. 4B, D) and for screws that were seen to penetrate the medial or lateral pedicle wall.
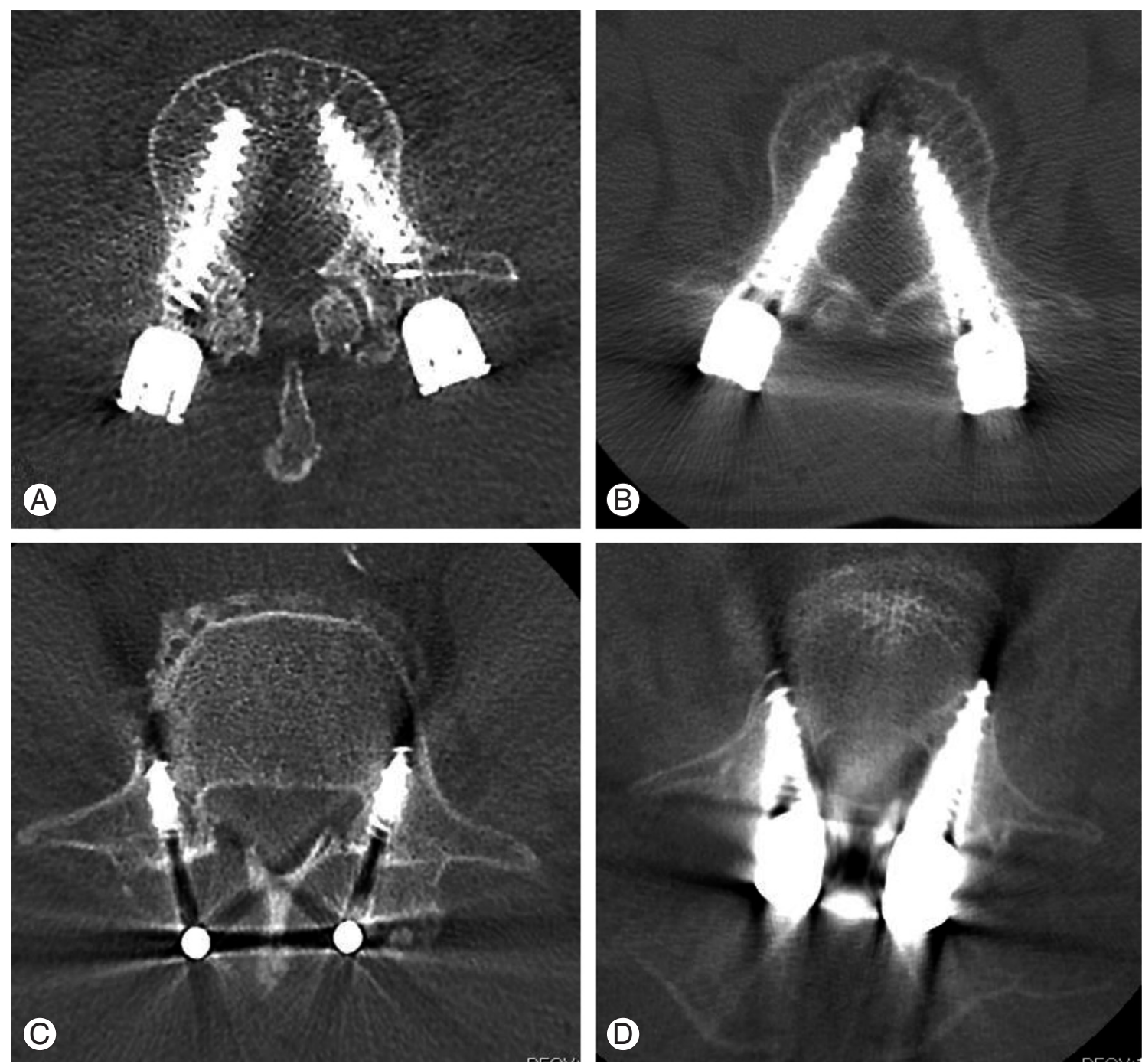

Fig. 4. Postoperative computed tomography of pedicle screw placement. Pedicle screw inserted conventionally or percutaneously in correct position (A), in contact with medial wall (B). Pedicle screw inserted with cortical bone trajectory in correct position (C), in contact with medial wall (D). 
Table 2. Operative variables, postoperative complications, and radiological findings

\begin{tabular}{|c|c|c|c|}
\hline Characteristic & M-TLIF $(n=10)$ & P-TLIF $(n=6)$ & CBT-TLIF $(n=10)$ \\
\hline Operative time (min) & $198 \pm 51$ & $243 \pm 26$ & $209 \pm 49$ \\
\hline Estimated blood loss (mL) & $429 \pm 289$ & $210 \pm 114$ & $188 \pm 167$ \\
\hline \multicolumn{4}{|l|}{ Complication during operation } \\
\hline Dural tear & 0 & 0 & 1 \\
\hline Pedicle fracture & - & - & $\begin{array}{c}2 \\
\text { CBT convert to conventional }\end{array}$ \\
\hline \multicolumn{4}{|l|}{ Lordosis angle of fusion level } \\
\hline Preoperative & $9.4 \pm 10.6$ & $12.0 \pm 10.8$ & $7.0 \pm 6.4$ \\
\hline Postoperative & $15.8 \pm 8.3^{\text {a) }}$ & $15.2 \pm 8.1$ & $10.9 \pm 2.9$ \\
\hline Final follow-up & $15.7 \pm 6.0$ & $11.0 \pm 7.7$ & $11.4 \pm 3.6$ \\
\hline \multicolumn{4}{|l|}{ Bone union at final follow-up } \\
\hline Fusion levels & 12 & 7 & 11 \\
\hline Complete & $10(83)$ & $7(100)$ & $10(91)$ \\
\hline Delayed & 2 & 0 & 1 \\
\hline
\end{tabular}

Values are presented as mean \pm standard deviation or number $(\%)$.

M-TLIF, transforaminal lumbar interbody fusion with minimally invasive pedicle screw insertion; P-TLIF, transforaminal lumbar interbody fusion with percutaneous pedicle screw insertion; CBT-TLIF, transforaminal lumbar interbody fusion with pedicle screw insertion with cortical bone trajectory.

${ }^{\text {a) }} p=0.01$ vs. preoperative angle by paired $t$-test.

\section{Statistical analyses}

Data are presented as means \pm standard deviations. The lordotic angles of each group were evaluated with the paired $t$-test preoperatively, postoperatively, and at final follow-up. The differences in each parameter among the three groups were evaluated by one-way analysis of variance followed by multiple comparison using Scheffe's method. All statistical analyses were performed using the Statistical Package for Biosciences software (SPBS, ver. 9.54) [12].

\section{Results}

Operative duration, EBL, complications, and radiological findings are presented in Table 2. The operative duration of P-TLIF was longer than that of M-TLIF $(p=0.06)$. Operative durations were not significantly different between M-TLIF and CBT-TLIF. EBL was significantly smaller in CBT-TLIF than in M-TLIF ( $p=0.03$ ), and smaller in PTLIF than in M-TLIF but not significantly so.

During CBT-TLIF, one case of dural tear and two cases of pedicle fracture at the insertion site on the facetectomy side occurred. The pedicle screw at each fracture site was inserted using a conventional trajectory, and fixation at the affected sites was stable in both cases.

Mean lordotic angle did not differ significantly among the three groups preoperatively but did increase postoperatively in all three groups. The increase in lordotic angle was statistically significant in M-TLIF ( $p=0.01$ ) but not in P-TLIF or CBT-TLIF. The postoperative and final lordotic angles were not significantly different among the three groups. Complete fusions were obtained in 10 of 12 levels (83\%) in 10 cases of M-TLIF, in seven levels (100\%) in six cases of P-TLIF, and in 10 of 11 levels (91\%) in 10 cases of CBT-TLIF.

Table 3 presents data on the postoperative positions of pedicle screws. Postoperative CT images revealed that $84.1 \%(37 / 44)$ of screws were positioned correctly with M-TLIF, 88.5\% (23/26) were positioned correctly with P-TLIF, and 90\% (38/42) were positioned correctly with CBT-TLIF. Seven screws in M-TLIF, one in P-TLIF, and four in CBT-TLIF were in contact with the medial wall of the affected pedicle. Two screws, both in P-TLIF, were in contact with the lateral wall of the pedicle. No screw was seen to penetrate the medial or lateral wall of the pedicle. 
Table 3. Postoperative position of pedicle screws as evaluated on CT images

\begin{tabular}{lccr} 
Characteristic & M-TLIF $(n=10)$ & P-TLIF $(n=6)$ & CBT-TLIF $(n=10)$ \\
No. of screws & 44 & 26 & 42 \\
Correct, $n(\%)$ & $37(84.1)$ & $23(88.5)$ & $38(90)$ \\
Contact with & & & 4 \\
Medial & 7 & 1 & 0 \\
Lateral & 0 & 0 & 0 \\
Incorrect & 0 & 0 & 0 \\
\hline
\end{tabular}

CT, computed tomography; M-TLIF, transforaminal lumbar interbody fusion with minimally invasive pedicle screw insertion; P-TLIF, transforaminal lumbar interbody fusion with percutaneous pedicle screw insertion; CBT-TLIF, transforaminal lumbar interbody fusion with pedicle screw insertion with cortical bone trajectory.

\section{Discussion}

In the present study, the perioperative results of TLIF using three different pedicle screw-insertion techniques were evaluated. CBT-TLIF resulted in a smaller intraoperative EBL volume and a shorter operative duration compared with conventional M-TLIF and P-TLIF. The fusion rate of the affected levels and the accuracy of screw positioning were similar in the three groups.

TLIF was first reported in 1998 by Harms and Jeszenszky [1]. The conventional open technique for pedicle screw insertion requires significant paraspinal muscle dissection and retraction to expose the screw entry point. To reduce damage to paraspinal muscles, minimally invasive approaches that preserve the lumbar spine musculature have been used [3,13-15]. Several studies have described advantages of M-TLIF with transpedicular screws, including reductions in blood loss and postoperative pain $[3,14,15]$. However, disadvantages of these techniques, including a steep learning curve, long operative duration, and technically demanding insertion of the pedicle screws, have been also reported $[10,16]$. The limited surgical field sometimes makes accurate insertion of pedicle screws difficult compared with a conventional open approach.

Thus, the percutaneous cannulated screw system, introduced by Magerl [17], was developed. Since then, the evolution of percutaneous pedicle screw instrumentation systems and expandable tubular retractors have contributed to the popularity of P-TLIF $[4,18]$. Minimally invasive lumbar fusion with pedicle screw insertion using a percutaneous system allows smaller incisions, less muscle stripping and blood loss, and excellent fusion and clinical outcomes $[4,19]$. On the other hand, several disadvantag- es have been reported, such as the learning curve [20], the accuracy of pedicle screw insertion [6], and complications associated with the use of Jamshidi needle or $\mathrm{K}$ wire as a guide for the pedicle screws [21]. In the present study, the duration of P-TLIF operations was longer than that of the other groups, suggesting the existence of a learning curve for this procedure, despite its lower EBL.

CBT is considered to have several advantages. First, the trajectory reduces the amount of paraspinal muscle exposure required. Second, the screw is placed from the inferior and medial border of the pedicle to the cranial and lateral corner of the posterior one-third of the vertebral body in a bicortical manner; thus, screws placed by CTB may provide stable fixation even in osteoporotic bone. However, there has been no report evaluating the clini$\mathrm{cal}$ and radiological results of CBT-TLIF. Post-CBT-TLIF lordotic angles were still being maintained a mean of 8 months following surgery, and the fusion rate of the affected levels and the accuracy of pedicle screw placement were similar to those of M-TLIF and P-TLIF procedures. We did experience two pedicle fractures with CBT. The fractures occurred at the caudal and facetectomy sides of the involved pedicles, and pedicle screws at these fracture sites were re-inserted using the conventional method to complete the fixation. The screws selected for the caudal and facetectomy sides were one size smaller than those we have recently used in the cranial side. Pedicle screws placed by CBT were intended to contact the medial wall of the pedicle, especially on the caudal side, but the screws did not penetrate the medial wall of the pedicle.

In a study by $\mathrm{Oh}$ et al. [22] on pedicle screw placement using percutaneous and open methods, the accuracy of pedicle wall penetration during screw fixation did not 
differ between the two techniques. In the present study, postoperative CT revealed the accuracy of pedicle screw insertion to be similar among the three groups. With CBT, the pedicle screw was inserted from medial to lateral; thus, the rate of medial perforation of the pedicle would be expected to be lower than with conventional or percutaneous pedicle screw insertion.

Limitations of this study include the small number of patients in each group and the short follow-up period; however, evaluation of larger numbers of patients with longer durations of follow-up is still ongoing and includes postoperative clinical results.

\section{Conclusions}

TLIF using CBT-inserted pedicle screws resulted in a smaller EBL volume and shorter operative duration than TLIF using conventionally or percutaneously inserted pedicle screws. CBT pedicle screw placement also resulted in rates of bone union, maintenance of lordotic angles, and accuracy of pedicle screw position that were similar to those of conventional or percutaneous methods.

\section{Conflict of Interest}

No potential conflict of interest relevant to this article was reported.

\section{References}

1. Harms JG, Jeszenszky D. Die posteriore, lumbale, interkorporelle Fusion in unilateraler transforaminaler Technik. Oper Orthop Traumatol 1998;10:90-102.

2. Rouben D, Casnellie M, Ferguson M. Long-term durability of minimal invasive posterior transforaminal lumbar interbody fusion: a clinical and radiographic follow-up. J Spinal Disord Tech 2011;24:288-96.

3. Foley KT, Holly LT, Schwender JD. Minimally invasive lumbar fusion. Spine (Phila Pa 1976) 2003;28:S26-35.

4. Harris EB, Massey P, Lawrence J, Rihn J, Vaccaro A, Anderson DG. Percutaneous techniques for minimally invasive posterior lumbar fusion. Neurosurg Focus 2008;25:E12.

5. Payer M. "Minimally invasive" lumbar spine surgery: a critical review. Acta Neurochir (Wien) 2011;153: 1455-9.

6. Kim MC, Chung HT, Cho JL, Kim DJ, Chung NS.
Factors affecting the accurate placement of percutaneous pedicle screws during minimally invasive transforaminal lumbar interbody fusion. Eur Spine J 2011;20:1635-43.

7. Tian NF, Wu YS, Zhang XL, Xu HZ, Chi YL, Mao FM. Minimally invasive versus open transforaminal lumbar interbody fusion: a meta-analysis based on the current evidence. Eur Spine J 2013;22:1741-9.

8. Santoni BG, Hynes RA, McGilvray KC, et al. Cortical bone trajectory for lumbar pedicle screws. Spine J 2009;9:366-73.

9. Meyerding HW. Spondyloptosis. Surg Gynaecol Obstet 1932;54:371-7.

10. Wang J, Zhou Y, Zhang ZF, Li CQ, Zheng WJ, Liu J. Comparison of one-level minimally invasive and open transforaminal lumbar interbody fusion in degenerative and isthmic spondylolisthesis grades 1 and 2. Eur Spine J 2010;19:1780-4.

11. Learch TJ, Massie JB, Pathria MN, Ahlgren BA, Garfin SR. Assessment of pedicle screw placement utilizing conventional radiography and computed tomography: a proposed systematic approach to improve accuracy of interpretation. Spine (Phila Pa 1976) 2004;29:767-73.

12. Murata K, Yano E. Medical statistics for evidencebased medicine with SPBS user's guide. Tokyo: Nankodo Publisher; 2002.

13. Rodriguez-Vela J, Lobo-Escolar A, Joven-Aliaga E, et al. Perioperative and short-term advantages of miniopen approach for lumbar spinal fusion. Eur Spine J 2009;18:1194-201.

14. Schwender JD, Holly LT, Rouben DP, Foley KT. Minimally invasive transforaminal lumbar interbody fusion (TLIF): technical feasibility and initial results. J Spinal Disord Tech 2005;18 Suppl:S1-6.

15. Holly LT, Schwender JD, Rouben DP, Foley KT. Minimally invasive transforaminal lumbar interbody fusion: indications, technique, and complications. Neurosurg Focus 2006;20:E6.

16. Villavicencio AT, Burneikiene S, Roeca CM, Nelson EL, Mason A. Minimally invasive versus open transforaminal lumbar interbody fusion. Surg Neurol Int 2010;1:12.

17. Magerl FP. Stabilization of the lower thoracic and lumbar spine with external skeletal fixation. Clin Orthop Relat Res 1984;(189):125-41.

18. Foley KT, Gupta SK. Percutaneous pedicle screw fixa- 
tion of the lumbar spine: preliminary clinical results. J Neurosurg 2002;97:7-12.

19. Park Y, Ha JW. Comparison of one-level posterior lumbar interbody fusion performed with a minimally invasive approach or a traditional open approach. Spine (Phila Pa 1976) 2007;32:537-43.

20. Lee JC, Jang HD, Shin BJ. Learning curve and clinical outcomes of minimally invasive transforaminal lumbar interbody fusion: our experience in 86 consecu- tive cases. Spine (Phila Pa 1976) 2012;37:1548-57.

21. Mobbs RJ, Raley DA. Complications with K-wire insertion for percutaneous pedicle screws. J Spinal Disord Tech 2014;27:390-4.

22. Oh HS, Kim JS, Lee SH, Liu WC, Hong SW. Comparison between the accuracy of percutaneous and open pedicle screw fixations in lumbosacral fusion. Spine J 2013;13:1751-7. 